\title{
Immunosignature: Serum Antibody Profiling for Cancer Diagnostics
}

\author{
Andrei I Chapoval ${ }^{1,2 \& *}$, J Bart Legutki ${ }^{2 \&}$, Philip Stafford ${ }^{2}$,Andrey V Trebukhov ${ }^{1}$, \\ Stephen A Johnston ${ }^{2}$, Yakov N Shoikhet ${ }^{3}$, Alexander F Lazarev ${ }^{4}$
}

\begin{abstract}
Biomarkers for preclinical diagnosis of cancer are valuable tools for detection of malignant tumors at early stages in groups at risk and screening healthy people, as well as monitoring disease recurrence after treatment of cancer. However the complexity of the body's response to the pathological processes makes it virtually impossible to evaluate this response to the development of the disease using a single biomarker that is present in the serum at low concentrations. An alternative approach to standard biomarker analysis is called immunosignature. Instead of going after biomarkers themselves this approach rely on the analysis of the humoral immune response to molecular changes associated with the development of pathological processes. It is known that antibodies are produced in response to proteins expressed during cancer development. Accordingly, the changes in antibody repertoire associated with tumor growth can serve as biomarkers of cancer. Immunosignature is a highly sensitive method for antibody repertoire analysis utilizing high density peptide microarrays. In the present review we discuss modern methods for antibody detection, as well as describe the principles and applications of immunosignature in research and clinical practice.
\end{abstract}

Keywords: Antibodies - autoantibodies - immunosignature - cancer - biomarkers - peptide microarray - diagnostics

Asian Pac J Cancer Prev, 16 (12), 4833-4837

\section{Background}

Cancer remains a constantly increasing cause of death worldwide. There were 14.1 million new cancer cases, 8.2 million cancer deaths and 32.6 million people living with cancer (within 5 years of diagnosis) in 2012 worldwide. The absolute number of new cancer cases in 2012 (14.1 million) was $40 \%$ higher as compare to 2000 (10.1 million) (Parkin et al., 2001; Ferlay et al., 2015). To date, despite various therapeutic intervention strategies directed to reduce cancer-related mortality all nations still face a significant cancer mortality rates. There is no doubt that the early diagnosis of tumors is the most promising approach to reduce the mortality caused by neoplastic diseases. Different approaches are used to find parameters/ biomarkers for a specific and sensitive diagnosis of early cancer stages. Presently there is a growing enthusiasm for applying proteomic approaches to the identification of serum biomarkers for evaluation of risk for cancer development, pre-symptomatic tumor diagnostics, guiding individualized therapy and prediction disease reoccurrence (Sawyers, 2008; Kulasingam et al., 2008).
For early detection to be effective and practical, biomarkers need to be available for non-invasive tests. Hallmarks of ideal biomarkers are stability, high specificity, and production in sufficient quantity to be detectable by a high throughput diagnostic. Current methods for protein biomarkers detection include direct analysis of soluble or cellular tumor-associated antigens (TAAs) such as p53, PSA, CEA, BRCA1/2, surviving, MUC1 and EGFR by 2D electrophoresis, ELISA, immunohistochemistry and other (Wang et al., 2012; Füzéry et al., 2013). These tests appear to be tumor specific but require established tumors for biopsy or production of sufficient concentration for detection in serum (Hori et al., 2011). In addition, these biomarkers are often unstable and monitoring them for specific changes can produce conflicting results for prognosis and prediction of the disease development (Fushiki et al., 2006; Anderson et al., 2013).

The demonstration of antibodies (Abs) against tumor associated antigens (TAA) have generated increasing interest as an alternative approach to circulating biomarkers to identify cancer at a localized and curable stage.

${ }^{1}$ Russian-American Anti-Cancer Center, Institute of Biomedicine, Altai State University, ${ }^{3}$ Department of Faculty Surgery, Altai State Medical University, ${ }^{4}$ Altai territory branch of Russian Cancer Research Center, Barnaul, Russia, ${ }^{2}$ Center for Innovations in Medicine, Biodesign Institute, Arizona State University, Tempe, AZ, USA ${ }^{\circledR}$ Equal contributions to this work *For correspondence: andreichapoval@gmail.com 


\section{Autoantibodies as Biomarkers of Cancer and Methods for their Detection}

Abs provide a first line of defense by detecting, neutralizing and clearing pathogens in an antigen (Ag)specific manner. There is growing number of evidence indicating that tumors express numerous types of antigens both wild-type (normal sequence but altered expression) and mutant (altered forms of existing proteins, or completely new sequences). Antigens associated with tumor development can stimulate B-cells to produce antibodies. This natural mechanism of signal amplification associated with antigenic stimulation of B-cells allow measuring the immune system reaction to minor molecular changes related to pathological processes. Analysis of serum antibodies repertoire is a logical path for early cancer diagnostic and prognosis. Examples of antibodies against TAAs and cancer biomarkers found in serum of cancer patients are presented in Table 1. The presence of antibodies against TAAs does not necessarily represent an anti-tumor defense mechanism but rather an immunological "fingerprint" of neoplastic diseases progression, as described in example of correlation between breast cancer and thyroid autoantibodies (Shi et al., 2014). It is not entirely clear how TAAs (often intracellular) trigger antibody production, but it has been suggested that overexpression and/or posttranslational modifications associated with aberrant cell death may enhance Ags immunogenicity under proinflammatory environment (Wu et al., 2001; Fernández Madrid 2005). Regardless of their biological role, antibodies that specifically recognize antigens derived from tumor cells may have a great potential as an early indicator of cancer development. The value of antibodies as cancer biomarkers is especially emphasized by their stability, specificity and rapid production in response to $\mathrm{Ag}$ encounter.

Current methods for identification of Abs against TAAs include cDNA expression libraries, phage display libraries, 2D western blots, 2D immunoaffinity and protein microarrays (Casiano et al., 2006; Tan et al., 2009; Heo et al., 2012). Despite a plethora of interesting and encouraging results generated by these methods, they are very time consuming and cannot be readily automatized for a clinical cancer diagnostic. The pros and cons of these methods are discussed below.

SEREX (Serological analysis of tumor antigens by recombinant cDNA expression cloning). Originally cDNA or cDNA phage libraries expression cloning was developed for identification of novel TAAs by screening patient sera against a cDNA expression library obtained from tumor tissue (Chen et al. 1997). For identification of Abs against TAAs the library is expressed in appropriate carrier and probed with patient and control sera. Using these approaches Abs profiles in sera of patients with lowgrade gliomas, prostate and lung cancer were analyzed (Wang et al., 2005; Gnjatic et al., 2009; Matsutani et al., 2012). One of the disadvantages of this method is that the library is usually generated from a single patient sample. Considering the heterogeneity of gene expression in different tumors a sample from one patient is not sufficient to explore whole verity of Abs produced against potential TAAs. In addition the expression libraries approach tends to catch only abundantly expressed $\mathrm{Ag}$ and not represent minor proteins well.

Another commonly used technique, termed SERPA (serological proteome analysis, for Ab screening, engages a laborious method of 2D electrophoresis of tumor cell lysates followed by blotting and subsequent probing with sera from healthy individuals or patients with cancer (Kellner et al., 2002; Farlow et al., 2010). Alternatively before electrophoresis and blotting tumor cells lysates can be depleted of proteins reactive with IgGs from sera of healthy controls on an immunoaffinity column, this method called MAPPing (multiple affinity protein profiling) (Caron, et al., 2007; Grandjean et al., 2013). These methods have been applied in the study of many cancers, such as melanoma, prostate, kidney and colorectal cancer (Klade et al., 2001; Wang et al., 2005; Hardouin et al., 2007). The drawbacks of these methods include the inherent limitations of 2D electrophoresis such as bias to abundant proteins and limitations for analysis of certain classes of proteins (e.g. membrane proteins) and difficulty in production of reproducible 2D gels (Tan et al., 2009; Heo et al.2012). In addition, these procedures are lengthy, costly and may be impacted by intra-laboratory variance.

The approaches that are more suitable for highthroughput and scalable include protein microarray and reverse-capture microarray. First, protein arrays are based on few TAA or hundreds to thousands of normal antigens which can be used for evaluation of the binding profile of various sera (MacBeath et al., 2000; Qin et al., 2014). On the other hand, the reverse capture microarrays represented by well-characterized, highly specific antiTAAs antibodies immobilized on a glass, than the arrays are loaded with tumor cell extracts and can be probed with labeled IgGs from cancer patients and appropriate controls

Table 1. Tumor antigens recognized by circulating antibodies

\begin{tabular}{llll}
\hline Protein & Cancer & Ab & \multicolumn{1}{c}{ Ref } \\
\hline HER2 & Breast & + & (Chapman et al. 2007) \\
p53 & Various & + & (Chapman et al. 2007, Chapman et al. 2008) \\
Cyclin B1 & Various & + & (Koziol et al. 2003, Zhang et al. 2003) \\
EGFR & Breast & + & (Azuma et al. 2014) \\
PTEN & Prostate & + & (O'Rourke et al. 2012) \\
MDM2 & Prostate & + & (Dai et al. 2014) \\
ALK & Lymphoma & + & (Pulford et al. 2000) \\
LKB1/STK11 & Kidney & + & (Scanlan et al. 1999) \\
BRAF & Melanoma & + & (Fensterle et al. 2004) \\
\hline
\end{tabular}


(Borrebaeck et al., 2009).

We know that there are cases when the true TAA is not present on a diagnostic microarray. The TAA specific antibodies often cross-react with other proteins and falsely identify an unrelated protein on the array (Kroening et al., 2012). For this reason and others, protein microarrays utilizing commercially available antibodies or known antigens have limited discovery power. Despite certain limitations, studies have demonstrated that microarrays have higher general predictive value than a single $\mathrm{Ab}$ marker (Zhong et al., 2004).

A major impediment to using known antigens to fish for tumor-specific Abs is the profound lack of candidates. We do not know enough about the process of tumor development to guess which antigens are most appropriate for broad cohort-based detection and discrimination. Many antigens reported in the literature are personal; finding ones that work well for multiple people requires expensive and complex large-scale studies. What if one could do without knowing the specific antigens? Imagine a sticky canvas against which one throws all serum antibodies. If this sticky canvas could sort the billions of antibodies into a coherent pattern, it would be a relatively simple process to examine which patterns correspond to personal components, which to unchanged "housekeeping" components, and which are common across many people with the same disease. This somewhat stochastic method may enable a diagnostic that encompasses high sensitivity, sufficient specificity, and the simplicity to create a clinical diagnostic.

\section{Immunosignature}

Recently a strategy using such a stochastic method was described (Stafford et al., 2012). An array of randomsequence peptides covalently bound to a glass slide was used to detect disease-dependent changes in the profile of circulating antibodies (Sykes et al., 2013). This strategy, termed immunosignatures, leverages the inherent flexibility in the range of antigens a single antibody can bind. The immunosignature peptides serve as partial or complete mimotopes of the antigen epitopes seen in disease. By providing multiple peptides, spread across combinatorial sequence space, a binding partner for an informative antibody can be found, even if an exact match for the epitope is absent. The use of multiple peptides significantly increases the predictive value of this assay as diagnostic test since it mimics a panel of potential TAAs necessary for a reasonable sensitivity and specificity. The present immunosignature microarrays are composed of 10,000 non-natural sequence 20mer peptides (Legutki et al., 2010) and produced by direct printing pre-synthesized peptides onto glass microscope slides. A second generation microarray containing 350,000 peptides averaging 11 amino acids long is being validated (Legutki et al., 2014). Peptides on the microarray are designed to cover as much of combinatorial sequence space as possible, rather than cover a specific pathogen or host proteome, allowing the same microarray to be used for any disease, whether chronic or infection, for mice, dogs and humans. By providing a set of ligands on which serum antibodies munosignature: Serum Antibody Profiling for Cancer Diagnostics.

can bind, the precise TAA does not need to be known to make a diagnosis. Frame shifts, chimeric proteins, or proteins which arise from genomic and transcriptional changes in cancers would not necessarily be included on a standard proteome array. Antibodies raised against these non-canonically encoded proteins could be captured on the immunosignature array (Kroening et al., 2012). The informative peptides (mimotopes) do not need to match a protein or TAA for reactive antibodies to be detected. Monoclonal antibodies raised against linear, conformational and carbohydrate epitopes have yielded specific immunosignatures on the arrays (Stafford et al., 2012). Notably, the larger the number of random-sequence peptides on the array, the higher the probability of actually finding a portion of a real linear epitope. 10,000 peptides is too few for that chance to occur often, but 350,000 11 mers contains a large portion of 4 mer and 5 mer space. Although not necessary for a clinical diagnostic, the presence of partial epitopes can be a useful research tool for creating therapeutics.

The assay principle of peptide microarrays is straight forward and similar to ELISA protocol. The arrays are manufactured on ISO-standard microscope slides allowing common microarray equipment to be used. The peptide arrays are first incubated with diluted patient or animal sera. After several washing steps, a fluorescent secondary antibody specific to the corresponding isotype is applied, enabling fluorescence-based detection. Conversion of these fluorescence images to numeric data is well established in the microarray field.

Data analysis and evaluation of results is the most important part of every microarray experiment. Initially fluorescent intensities are normalized to the array and subjected to a quality control algorithm. Quality control identifies arrays with gross or even subtle image artifacts without the need to examine each image by hand. High quality replicates are averaged and used to derive an immunosignature. Informative peptides comprising the disease immunosignature are selected using a two tailed t-Test ( $p$ values typically < 10-7) followed by fold change cutoffs and pattern-matching to heighten contrast between case and control. Robustness of the immunosignature is initially evaluated by training a machine learning algorithm, typically a support vector machine (SVM), on a training set of patients to determine the cross-validation error. Predictive accuracy, specificity and sensitivity of the immunosignature are determined by using the trained algorithm to classify an independent test set of patients not included in feature selection. Once a validated set of peptides that sensitively and specifically detects and discriminates a disease is chosen, the feature selection is no longer needed. If, however, additional diseases are added to a diagnostic panel, one needs to ensure that these peptides can discriminate against the new disease. Without examining patient sera, one cannot know a priori whether the new disease may have some immunological overlap with the current panel of detectable diseases.

To date, immunosignatures have been used to classify over 40 different diseases in both humans and dogs (published and unpublished studies). Accuracy in predicting an independent test set typically exceeds $94 \%$ 
(Hughes et al., 2012; Stafford et al., 2014). A published study investigating the immunosignature of brain cancer, specific types of cancer were readily distinguished including the aggressive glioblastoma multiforme (GBM) (Hughes et al., 2012). In GBM patients, the O6-methy-guanine-DNA methyltransferase methylation promoter (MGMT) is correlated with survival following temozolomide treatment. This single epigenetic change has a detectable effect on the immunosignature indicating that immune profiles can mimic existing molecular biomarkers, but can also extend their capability (Hughes et al., 2012). The organ in which the cancer originates can be distinguished; the immunosignatures of breast and brain cancer are unique to their cohorts (Hughes et al., 2012). Within an organ, diseases produce different immunosignatures. Pancreatic cancer is distinguishable from pancreatitis and PanIN, an early stage of pancreatic cancer development (Kukreja et al., 2012). Detection of PanIN's indicate that the immunosignature may have utility in detecting cancer early, before metastasis and when treatment can be effective. Alzheimer's Disease (AD) is another chronic disease that develops over decades. The immunosignature can distinguish $\mathrm{AD}$ patients from age matched control and non-AD caused dementia (Restrepo et al., 2013). An example for the early detection of chronic disease is given in a mouse AD model. Transgenic mice bearing presenilin 1 and chimeric amyloid beta can be distinguished from the parental wildtype strain prior to the development of senile plaques and advanced cognitive degredation (Restrepo, Stafford, and Johnston 2013). In a murine model of influenza infection, the immunosignature can detect infection 3 days earlier than an ELISA (Restrepo et al., 2013).

In order to facilitate health monitoring, which is what early detection of cancer really is, one needs a robust, repeatable, inexpensive and simple assay that is not subject to intra-laboratory variance. Microarrays can be very reproducible provided that the sample preparation does not impact the results. RNA quality, as an example, can profoundly change the gene expression profile. Extraction of tumor cells from within vs. without a tumor mass can also change these expression profiles. Circulating antibodies are very stable, are generally at similar concentrations from person to person, and have a distinct advantage of needing no sample preparation.

\section{Acknowledgements}

This paper was supported by grant from the Ministry of Education and Science of the Russian Federation within the framework of the competitive part of the state task in scientific research "Altai State University" Project Code: 303. S.A.J. is a co-founder of HealthTell, Inc., which develops immunosignature peptide microarray for cancer diagnostic. J.B.L is employed by HealthTell, Inc. The authors have no other relevant affiliations or financial involvement with any organization or entity with a financial interest in or financial conflict with the subject matter or materials discussed in the manuscript apart from those disclosed.

\section{References}

Anderson NL, Ptolemy AS, Rifai N (2013). The riddle of protein diagnostics: future bleak or bright? Clin Chem, 59, 194-7.

Azuma K, Komatsu N, Hattori S, et al (2014). Humoral immune responses to EGFR-derived peptides predict progression-free and overall survival of non-small cell lung cancer patients receiving gefitinib. PLoS One, $9,86667$.

Borrebaeck CA, Wingren C (2009). Design of high-density antibody microarrays for disease proteomics: key technological issues. J Proteomics, 72, 928-5.

Caron M, Choquet-Kastylevsky G, Joubert-Caron R (2007). Cancer immunomics using autoantibody signatures for biomarker discovery. Mol Cell Proteomics, 6, 1115-2.

Casiano CA, Mediavilla-Varela M, and Tan EM (2006). Tumorassociated antigen arrays for the serological diagnosis of cancer. Mol Cell Proteomics, 5, 1745-9.

Chapman CJ, Murray A, McElveen JE, et al (2008). Autoantibodies in lung cancer: possibilities for early detection and subsequent cure. Thorax, 63, 228-3.

Chapman C, Murray A, Chakrabarti J, et al (2007). Autoantibodies in breast cancer: their use as an aid to early diagnosis. Ann Oncol, 18, 868-3.

Chen YT, Scanlan MJ, Sahin U, et al (1997). A testicular antigen aberrantly expressed in human cancers detected by autologous antibody screening. Proc Natl Acad Sci U S A, 94, 1914-8.

Dai L, Li J, Ortega R, et al (2014). Preferential autoimmune response in prostate cancer to cyclin B1 in a panel of tumorassociated antigens. J Immunol Res, 2014, 827827.

Farlow EC, Vercillo MS, Coon JS, et al (2010). A multi-analyte serum test for the detection of non-small cell lung cancer. Br J Cancer, 103, 1221-8.

Fensterle, J., J. C. Becker, T. Potapenko, et al (2004). B-Raf specific antibody responses in melanoma patients. $B M C$ Cancer, 4, 62.

Ferlay J, Soerjomataram I, Dikshit R, et al (2015). Cancer incidence and mortality worldwide: sources, methods and major patterns in GLOBOCAN 2012. Int J Cancer, 136, 359-6.

Fernández Madrid F (2005). Autoantibodies in breast cancer sera: candidate biomarkers and reporters of tumorigenesis. Cancer Lett, 230, 187-8.

Fushiki T, Fujisawa H, Eguchi S (2006). Identification of biomarkers from mass spectrometry data using a "common" peak approach. BMC Bioinformatics, 7, 358 .

Füzéry AK, Levin J, Chan MM, Chan DW (2013). Translation of proteomic biomarkers into FDA approved cancer diagnostics: issues and challenges. Clin Proteomics, 10, 13.

Gnjatic S, Wheeler C, Ebner M, et al (2009). Seromic analysis of antibody responses in non-small cell lung cancer patients and healthy donors using conformational protein arrays. $J$ Immunol Methods, 341, 50-8.

Grandjean M, Dieu M, Raes M, Feron O (2013). A new method combining sequential immunoaffinity depletion and differential in gel electrophoresis to identify autoantibodies as cancer biomarkers. J Immunol Methods, 396, 23-2.

Hardouin J, Lasserre JP, Sylvius L, Joubert-Caron R, and Caron M (2007). Cancer immunomics: from serological proteome analysis to multiple affinity protein profiling. Ann N Y Acad Sci, 1107, 223-0.

Heo CK, Bahk YY, Cho EW (2012). Tumor-associated autoantibodies as diagnostic and prognostic biomarkers. BMB Rep, 45, 677-5.

Hori SS, Gambhir SS (2011). Mathematical model identifies blood biomarker-based early cancer detection strategies and limitations. Sci Transl Med, 3, 109-6 
Hughes AK, Cichacz Z., Scheck A, et al (2012). Immunosignaturing can detect products from molecular markers in brain cancer. PLoS One , 7, 40201.

Kellner R, Lichtenfels R, Atkins D, et al (2002). Targeting of tumor associated antigens in renal cell carcinoma using proteome-based analysis and their clinical significance. Proteomics, 2, 1743-1.

Klade CS, Voss T, Krystek E, et al (2001). Identification of tumor antigens in renal cell carcinoma by serological proteome analysis. Proteomics, 1, 890-8.

Koziol JA, Zhang JY, Casiano CA, et al (2003). Recursive partitioning as an approach to selection of immune markers for tumor diagnosis. Clin Cancer Res, 9, 5120-6.

Kroening K., Johnston SA, Legutki JB (2012). Autoreactive antibodies raised by self derived de novo peptides can identify unrelated antigens on protein microarrays. Are autoantibodies really autoantibodies? Exp Mol Pathol, 92, 304-1.

Kukreja M, Johnston SA, Stafford P (2012). Comparative study of classification algorithms for immunosignaturing data. BMC Bioinformatics, 13, 139.

Kulasingam V, Diamandis EP (2008). Strategies for discovering novel cancer biomarkers through utilization of emerging technologies. Nat Clin Pract Oncol, 5, 588-9.

Legutki JB, Magee DM, Stafford P, and Johnston SA (2010). A general method for characterization of humoral immunity induced by a vaccine or infection. Vaccine, 28, 4529-7.

Legutki JB, Zhao ZG, Greving M, et al (2014). Scalable highdensity peptide arrays for comprehensive health monitoring. Nat Commun, 5, 4785.

MacBeath G, Schreiber SL (2000). Printing proteins as microarrays for high-throughput function determination. Science, 289, 1760-3.

Matsutani T, Hiwasa T, Takiguchi M, et al (2012). Autologous antibody to src-homology 3-domain GRB2-like 1 specifically increases in the sera of patients with low-grade gliomas. $J$ Exp Clin Cancer Res, 31, 85.

O'Rourke D J, DiJohnson DA, Caiazzo RJ, et al (2012). Autoantibody signatures as biomarkers to distinguish prostate cancer from benign prostatic hyperplasia in patients with increased serum prostate specific antigen. Clin Chim Acta, 413, 561-7.

Parkin DM, Bray F, Ferlay J, Pisani P (2001). Estimating the world cancer burden: Globocan 2000. Int J Cancer, 94, 153-6.

Pulford, K, Falini B, Banham AH, et al (2000). Immune response to the ALK oncogenic tyrosine kinase in patients with anaplastic large-cell lymphoma. Blood, 96, 1605-7.

Qin JJ, Wang XR, Wang P, et al (2014). Mini-array of multiple tumor-associated antigens (TAAs) in the immunodiagnosis of esophageal cancer. Asian Pac J Cancer Prev, 15, 2635-0.

Restrepo L, Stafford P, Johnston SA (2013). Feasibility of an early Alzheimer's disease immunosignature diagnostic test. J Neuroimmunol, 254, 154-0.

Sawyers CL (2008). The cancer biomarker problem. Nature, 452, 548-2.

Scanlan MJ, Gordan JD, Williamson B, et al (1999). Antigens recognized by autologous antibody in patients with renal-cell carcinoma. Int J Cancer, 83, 456-4.

Shi XZ, Jin X, Xu P, Shen HM (2014). Relationship between breast cancer and levels of serum thyroid hormones and antibodies: a meta-analysis. Asian Pac J Cancer Prev, 15, 6643-7.

Stafford P, Cichacz Z, Woodbury NW, Johnston SA (2014). Immunosignature system for diagnosis of cancer. Proc Natl Acad Sci U S A, 111, 3072-0.

Stafford P, Halperin R, Legutki JB, et al (2012). Physical munosignature: Serum Antibody Profiling for Cancer Diagnostics. characterization of the "immunosignaturing effect. Mol Cell Proteomics, 11, 111.

Sykes KF, Legutki JB, Stafford P (2013). Immunosignaturing: a critical review. Trends Biotechnol, 31, 45-1.

Tan HT, Low J, Lim SG, Chung MC (2009). Serum autoantibodies as biomarkers for early cancer detection. FEBS J, 276, 6880-4.

Wang X, Yu J, Sreekumar A, et al (2005). Autoantibody signatures in prostate cancer. $N$ Engl J Med, 353, 1224-5.

Wang YQ, Zhang HH, Liu CL, et al (2012). Correlation between auto-antibodies to survivin and MUC1 variable number tandem repeats in colorectal cancer. Asian Pac J Cancer Prev, 13, 5557-2.

Wu X, Molinaro C, Johnson N, Casiano CA (2001). Secondary necrosis is a source of proteolytically modified forms of specific intracellular autoantigens: implications for systemic autoimmunity. Arthritis Rheum, 44, 2642-2.

Zhang JY, Casiano CA, Peng XX, et al (2003). Enhancement of antibody detection in cancer using panel of recombinant tumor-associated antigens. Cancer Epidemiol Biomarkers Prev, 12, 136-3.

Zhong L, Peng X, Hidalgo GE, et al (2004). Identification of circulating antibodies to tumor-associated proteins for combined use as markers of non-small cell lung cancer. Proteomics, 4, 1216-5. 